\title{
miR-26b promoter analysis reveals regulatory mechanisms by lipid-related transcription factors in goat mammary epithelial cells
}

\author{
Hui Wang, ${ }^{*}$ Jun Luo, ${ }^{* 1}$ Qiuya He, ${ }^{*}$ Dawei Yao,† Jiao Wu, ${ }^{*}$ and Juan J. Loor \\ *Shaanxi Key Laboratory of Molecular Biology for Agriculture, College of Animal Science and Technology, Northwest A\&F University, Yangling, \\ Shaanxi, 712100, P. R. China \\ †Tianjin Animal Science and Veterinary Research Institute, 17 km. Jin-Jing Road, Xi-Qing District, Tianjin, 300381, P. R. China \\ ¥Mammalian NutriPhysioGenomics, Department of Animal Sciences and Division of Nutritional Sciences, University of Illinois, Urbana 61801
}

\begin{abstract}
MicroRNA (miRNA) regulate protein abundance and control diverse aspects of cellular processes and biological functions associated with lipid metabolism. MiR-26b and its host gene CTDSP1 regulate triacylglycerol synthesis by synergistically suppressing the insulin-induced gene 1 (INSIG1); however, the direct regulators of miR-26b expression remain unknown. In the present study, we characterized the activity of a novel putative promoter region in miR-26b. Results revealed that promoter activity and miR-26b expression are dynamically regulated by different transcription factors including peroxisome proliferator-activated receptor gamma (PPARG), sterol regulatory element binding transcription factor 1 (SREBF1), and liver $\mathrm{X}$ receptor $\alpha(L X R \alpha)$. Two binding sites for the SREBF1 (SRE1 and SRE3) and the PPARG (peroxisome proliferator response element 1 and 2; PPRE1 and PPRE2), respectively, were identified in the miR-26b promoter, which demonstrated that those binding sites are responsible for the activation by PPARG and SREBF1. In silico analysis and site-directed mutagenesis of LXR $\alpha$ binding elements (LXRE) and SREBF1 binding elements (SRE) revealed that the effects of Ad-LXR $\alpha+$ T0901317 requires the presence of SRE, whereas potential LXRE had no effects on miR-26b expression. This suggested that regulation of miR-26b by LXR $\alpha$ is indirectly via an SRE, and miR-26b is regulated by transcription factors dually through DNA methylation and directly through binding to its promoter, all of which implies that regulation of miR-26b in ruminant mammary epithelial cells results from various mechanisms. In conclusion, we demonstrate a novel dual-regulatory mechanism whereby transcription factors regulate the expression of miR-26b. Overall, these findings contribute to our understanding of the interactions between
\end{abstract}

Received December 12, 2016.

Accepted March 29, 2017.

${ }^{1}$ Corresponding author: luojun@nwsuaf.edu.cn specific promoter elements and the control of transcription and translation of miRNA.

Key words: miR-26b promoter, transcription factor, DNA methylation, ruminant mammary epithelial cell

\section{INTRODUCTION}

The microRNA (miRNA) are a subclass of noncoding RNA that trigger mRNA degradation or block mRNA translation in a sequence-dependent manner (Filipowicz et al., 2008). The miRNA sequences are dispersed throughout the genome and are classified as intergenic (between genes) or intronic (embedded into a gene) miRNA (Monteys et al., 2010). Almost 40\% of miRNA are located within intronic regions of proteincoding transcriptional units (Kim et al., 2009). Analysis of 175 human miRNA across 24 different human organs reveals that the expression of intronic miRNA coincides largely with the transcription of their host transcriptional units (Baskerville and Bartel, 2005), indicating that the intronic miRNA and their host genes may be co-regulated and are generated from a common precursor transcript.

Over the course of evolution, the genomic loci of the miR-26 family have been localized to the introns of genes coding for the proteins of the carboxy-terminal domain RNA polymerase II polypeptide A small phosphatase family. The miR-26 family consists of miR-26a and miR-26b; they are transcribed from 3 genomic loci, miR-26a-1, miR-26a-2, and miR-26b, which reside in the introns of genes coding for CTDSPL, CTDSP2, and CTDSP1 proteins, respectively. The miR-26 family is known to play crucial roles in tumorigenesis by targeting critical regulators involved in development $(\mathrm{Fu}$ et al., 2014), cell type, and differentiation (Dill et al., 2012). Under physiological and pathological conditions, Zhu et al. (2012) reported that miR-26b is expressed concomitantly with its host genes, and they cooperate to block the G1/S-phase transition by synergistically activating the pRb protein (Zhu et al., 2012). We also demonstrated recently that miR-26b and CTDSP1 
synergistically regulate the triacylglycerol synthesis in goat mammary epithelial cells (GMEC; Wang et al., 2016). Although many functional studies are available for miR-26b, the transcriptional regulation of miR-26b has not been studied.

Peroxisome proliferator-activated receptor gamma (PPARG) and liver X receptor $\alpha(\mathbf{L X R} \boldsymbol{\alpha})$ are ligandactivated transcription factors that play crucial roles in the regulation of lipid synthesis (Wang et al., 2012; Shi et al., 2013), sterol regulatory element binding transcription factor 1 (SREBF1) is a membrane-bound transcription factor that activates genes encoding enzymes required for synthesis of triacylglycerol and cholesterol (Fan et al., 2008). The activation of these 3 key transcription regulators induces lipid synthesis by modulating genes that contain a peroxisome proliferator response element (PPRE), LXR response element (LXRE), and sterol response element (SRE) in the promoter, respectively. For instance, in goats and mice, SREBF1 regulates fatty acid synthase (FASN) expression, a central enzyme in the de novo fatty acid biosynthesis pathway, through 2 SRE (Wang and Sul, 1997; Li et al., 2015a); in GMEC, LXR can regulate promoter activity of FASN (Li et al., 2015b), SREBP1 (Xu et al., 2016), and stearoyl-CoA desaturase 1 (SCD1; Yao et al., 2016) through direct and indirect mechanisms. Activation of PPARG in GMEC in response to the chemical ligand rosiglitazone (ROSI) increased the expression of many lipid-related genes and increased lipid accumulation through the direct regulation of adipose differentiation-related protein (ADRP) by binding PPRE on its promoter (Yu et al., 2015).

Previous in silico analysis of the miR-107 promoter sequence to search for transcription factor binding sites revealed the presence of binding sites for transcription factors such as myogenin, CCAAT-enhancer-binding protein $(\mathrm{C} / \mathrm{EBP})$, specificity protein 1 (SP1), cAMPresponse element binding protein (CREBP), peroxisome proliferator-activated receptor alpha (PPARA), and SREBP (Daimiel-Ruiz et al., 2015). When rats were treated with ROSI, expression of 28 miRNA in the cerebral cortex was altered significantly, and in silico analysis indicated the existence of 1 to 5 PPRE in the putative promoter regions of these miRNA. Co-transfection with a PPARG constitutively expressing vector significantly induced activity of miR-145 and miR-329 promoter vectors (each have 4 PPRE), a response that was curtailed by point mutations of the PPRE in the promoters (Dharap et al., 2015).

Although transcriptional regulation of miRNA has been investigated in humans and mice, the regulation of miRNA by transcription factors in ruminants is nonexistent. In fact, whether and how transcription factors control the expression of miRNA in the mammary gland remains to be determined. This study was designed to clone and characterize the goat miR-26b promoter, and investigate mechanisms of transcriptional regulation by the 3 key lipid-related transcription factors (PPARG, SREBP, and LXR $\alpha$ ) in goat mammary cells. Our findings illustrated that PPARG, SREBP, and LXR $\alpha$ regulate the expression of miR-26b by binding PPRE and SRE on its promoter, respectively. These data are useful for the determination of lipid synthesis regulatory networks in dairy ruminants.

\section{MATERIALS AND METHODS}

\section{Cloning of the miR-26b Promoter Sequence and Bioinformatics Analysis}

Goat miR-26b promoter sequence was amplified from goat blood genomic DNA using PrimeSTAR Max DNA Polymerase (Takara Bio Inc., Otsu, Japan) base on the Yunnan black goat genome (Corcoran et al., 2009). The forward primer (TATTAATATTTTAAAAATGTGAACA) and the reverse primer (TTTCAAGGCTCCCCCTTAAA) were used for miR26b promoter PCR amplification. Amplified products were cloned into the pMD19-T vector (Takara Bio Inc.) and sequenced by Invitrogen (Shanghai, China).

The promoter region was predicted using Promoter 2.0 (http://www.cbs.dtu.dk/services/Promoter/). The putative transcription factor binding sites were initially predicted using the following online tools: MatInspector (http://www.genomatix.de/), JASPAR 2016 server (http://jaspar.genereg.net/). AliBata 2.1 (http://www.gene-regulation.com/pub/programs.

html), TFSEARCH (http://diyhpl.us/ bryan/irc/ protocol-online/protocol-cache/TFSEARCH.html),

and the TRED (Transcriptional regulatory element database, https://cb.utdallas.edu/cgi-bin/TRED/tred. cgi? process $=$ searchTFGeneForm).

\section{Plasmid Construction}

The resulting fragment of the miR-26b region was employed in PCR to introduce KpnI and HindIII restriction sites with the cloning primer pairs listed in Table 1. The PCR product was digested with KpnI and HindIII and inserted into the upstream of the luciferase gene using the pGL-3 basic vector (Promega, Madison, WI).

To generate mutation plasmids, an overlap PCR strategy was used to introduce site-directed mutations in the binding sites for PPRE, SRE, and LXRE in the miR-26b promoter region. First, to generate partially 
Table 1. Primers used for isolation and site-directed mutagenesis of miR-26b promoter

\begin{tabular}{|c|c|c|c|}
\hline Primer name & Primer & Primer sequence $\left(5^{\prime} \text { to } 3^{\prime}\right)^{1}$ & $\begin{array}{c}\text { Binding } \\
\text { region } \\
\text { (bp) }\end{array}$ \\
\hline Cloning primer & Forward & GG $\boldsymbol{G} \boldsymbol{G} \boldsymbol{T A} \boldsymbol{C} \boldsymbol{C T A T T A A T A T T T T A A A A A T G T G A A C A ~}$ & $-1,018$ \\
\hline \multirow{22}{*}{$\begin{array}{l}\text { Site-directed mutagenesis } \\
\text { primer }\end{array}$} & SRE1 & AGCTGCCAGTC & -269 \\
\hline & SRE1-mut & GAGAAGACCGAGAGGCCGACAATCCCCG & -269 \\
\hline & SRE1-anti-mut & CCAAAGGTCGGGGATTGTCGCCCTCTC & -269 \\
\hline & SRE3 & GTGAGGAGAG & -529 \\
\hline & SRE3-mut & $\begin{array}{l}\text { GTGGCCGGGCGCCCGTTGGGAAAAGGTC } \\
\text { CGCCCGGTGAGGAGAGGTCG }\end{array}$ & -529 \\
\hline & SRE3-anti-mut & GGTGCCCCTGCCGACCTTTTCCCAACGGG & -529 \\
\hline & SRE4 & CTGGGGTGGG & -568 \\
\hline & SRE4-mut & GAAAACCAGGTGGTTTGTGTTGGCATG & -568 \\
\hline & PPRE2-mut & GTGTGGATCAACACAGTTTTTTGGAAG & -396 \\
\hline & PPRE2-anti-mut & GCTGCACTTCCAAAAAACTGTGTTGATC & -396 \\
\hline & PPRE3 & TTAGGGGAAAGGGGC & -461 \\
\hline & PPRE3-mut & GCTCCGGGTTTGTGGGAAATTGGCCGAG & -461 \\
\hline & PPRE3-anti-mut & GCAGTCTCGGCCAATTTCCCACAAACCC & -461 \\
\hline & PPRE4 & GCAGAGGAGGGGGCA & -728 \\
\hline & PPRE4-mut & CGGGTGGGAAGCCTAGGAGTTGGCATGGG & -728 \\
\hline & PPRE4-anti-mut & CCTTTTTCCCATGCCAACTCCTAGGCTTCC & -728 \\
\hline & LXRE1 & CTCGGGGCACTCCCGGGCG & -220 \\
\hline & LXRE1-mut & GAGTCGCGCTGCCGAGGGGCCATCCCGGAAGCGGG & -220 \\
\hline & LXRE1-anti-mut & GCCCGCGCCCGCTTCCGGGATGGCCCCTCGGCAG & -220 \\
\hline & LXRE2 & GCAGGGGCACCCCGGGGCG & -515 \\
\hline & LXRE2-mut & GTGAGGAGAGGTCGGTGGGGGCAAACCGGAACGGACAG & -515 \\
\hline & LXRE2-anti-mut & GTTCCAGCTGTCCGTTCCGGTTTGCCCCCACCGAC & -515 \\
\hline
\end{tabular}

${ }^{1}$ Mutation letters are enhanced in bold fonts. Restriction enzyme sites are shown in italic and enhanced in bold fonts.

${ }^{2}$ Number of nucleotides upstream $(-)$ and downstream $(+)$ from the transcriptional start site.

overlapping fragments, the site-directed mutagenesis primers listed in Table 1 were used with the wild-type insert as template. Then, the 2 DNA fragments with the designated mutations were gel-purified and used in a second PCR as templates to generate a third DNA fragment. The PPRE, SRE, and LXRE mutants were constructed using wild-type constructs as templates. The final overlapping fragments were then digested with $K p n I$ and HindIII to generate mutant constructs. The presence of the correct insert of all the mutation plasmids were confirmed by automated DNA sequencing.

\section{Cell Culture and Treatment}

Goat primary epithelial cells were obtained from peak lactation Xinong Saanen dairy goats and the culture procedures were according to our previous publications (Wang et al., 2010; Shi et al., 2014). For the miR-26b promoter activity assay, the cells were seeded in 48-well plates (Corning Inc., Corning, NY) the day before transfection at a density of $5.0 \times 10^{4}$ cells per well. Then, the GMEC were transiently transfected with a Transfection Reagent (Lipofectamine 2000, Life Technologies, Waltham, MA) according to the manufacturer's protocols. Each well contained $300 \mathrm{ng}$ of total DNA and $10 \mathrm{ng}$ of the internal control vector pRL-TK (Promega, Madison, WI). After transfection for $12 \mathrm{~h}$, cells were treated with adenovirus containing peroxisome proliferator-activated receptor-gamma (AdPPARG) or adenovirus containing liver-X-receptor $\alpha$ (Ad-LXR $\alpha)$. At $12 \mathrm{~h}$ posttreatment, cell culture medium was changed with starvation medium free of fetal bovine serum, and incubated for $12 \mathrm{~h}$. T0901317 (T09, a specific agonist of LXR) and ROSI (a specific agonist of PPARG) were added to the fetal bovine serum-free culture medium before incubation of cells for $12 \mathrm{~h}$, respectively. The concentration of T09 was $1 \mu M$ and ROSI was $50 \mu M$ according to our previous experiments (Yu et al., 2015; Wang et al., 2016). 
For miRNA expression analysis, the GMEC were seeded in 12-well plates and cultured until 70 to $80 \%$ confluence. Cells were then treated with Ad-PPARG or Ad-LXR $\alpha$ for $12 \mathrm{~h}$, and then cell culture medium was changed with starvation medium free of fetal bovine serum followed by incubation for another $12 \mathrm{~h}$. The ROSI, T09, and different concentration of dimethyl sulfoxide (control groups) were added to the fetal bovine serum-free culture medium before incubation of cells for $24 \mathrm{~h}$, respectively. An independent study was done using adenovirus containing sterol regulatory element binding proteins (Ad-SREBF1), and cells were harvested after $48 \mathrm{~h}$ of incubation.

\section{Dual-Luciferase Reporter Assays}

A sufficient volume of PBS was applied to wash the cells from the surface of the culture vessel, and cell lysates were harvested with $1 \times$ passive lysis buffer. After first adding $100 \mu \mathrm{L}$ of luciferase assay reagent (LAR) into a 96-microwell plate, $20 \mu \mathrm{L}$ of cell lysate was transferred into the tube to determine the activity of firefly luciferase in PGL3 $\left(\boldsymbol{h l u c}^{+}\right)$. Then, $100 \mu \mathrm{L}$ of Stop \& Glo Reagent was added into the plates to measure the activity of Renilla luciferase in pRL-TK (hRluc) with the dual-luciferase reporter assay system according to the manufacturer's protocols (Promega, Madison, WI) by a Varioskan flash (Thermo Fisher Scientific, Waltham, MA). The ratio hluc $/ h R l u c$ was used to determine the relative luciferase activity.

\section{Quantitative Real-Time PCR Analysis}

Quantitative real-time PCR was performed as previously described (Kang et al., 2012; Wang et al., 2016). In short, total RNA was extracted from the GMEC using Trizol reagent (Invitrogen, Waltham, MA) and reverse transcribed using the PrimeScript RT Reagent Kit (Perfect Real Time, Takara, Japan). The resulting cDNA was amplified by RT-PCR using the S-Poly (T) assay kit. The miRNA expression was calculated with the relative quantification method using the $5 \mathrm{~S}$ rRNA as reference gene. The sequences for all primers used in quantitative PCR were reported previously (Wang et al., 2016). Relative expression was calculated using the relative quantification $\left(2^{-\Delta \Delta \mathrm{CT}}\right)$ method.

\section{Quantitation of DNA Methylation Via Bisulfite Genomic Sequencing PCR}

The GMEC were treated as described for miRNA expression analysis assay and collected $48 \mathrm{~h}$ later. Genomic DNA was extracted from the GMEC using an Axygen
Genomic DNA purification kit (Axygen Biotechnology, Hangzhou, China). Quantitative DNA methylation analysis of the miR-26b promoter region was performed using bisulfite sequencing PCR (BSP). Genomic DNA (450 ng) was treated with sodium bisulfite using an EZ DNA Methylation-Gold kit (Zymo Research, Orange, CA) according to the manufacturer's protocol (https:// www.zymoresearch.com/epigenetics/dna-methylation/ bisulfite-conversion/ez-dna-methylation-gold-kit). The miR-26b promoter was amplified from modified genomic DNA by PCR. The primers used in BSP were designed using MethPrimer tool (http://www.urogene. org/cgi-bin/methprimer/methprimer.cgi) and are listed in Table 1. The PCR products were subcloned into PMD-19T vectors (Takara Bio Inc., Otsu, Japan) and transformed into Top 10 E. coli (Tiagen, Beijing, China). Candidate plasmid clones were sequenced at a commercial laboratory (Genscript Company, Nanjing, China).

\section{Chromatin Immunoprecipitation Assay}

Chromatin immunoprecipitation (ChIP) assays were performed by using the ChIP assay kit (Beyotime, Shanghai, China) according to the manufacturer's protocols (http://www.beyotime.com/product/P2078. htm). Briefly, GMEC were seeded onto $100 \mathrm{~mm} \times 20$ mm cell culture dishes and cultured until 90 to $100 \%$ confluence. Then, cells were fixed with $1 \%$ formaldehyde for $10 \mathrm{~min}$ at $37^{\circ} \mathrm{C}$. The cells were washed with cold PBS and cell lysates were sonicated to produce chromatin fragments averaging 200 to 1,000 bp. The fragmented chromatin was then added to ChIP dilution buffer (Beyotime) and the samples were incubated overnight at $4^{\circ} \mathrm{C}$ with anti-SREBF1 (Abcam, Cambridge, MA) and normal mouse IgG (Millipore, Darmstadt, Germany) antibodies. The resultant immune complexes were precipitated with protein $\mathrm{A}+\mathrm{G}$ agarose/Salmon Sperm DNA (Beyotime) and washed with wash buffer (Beyotime). Finally, the DNA-protein crosslinks were reversed by incubating the samples in $5 \mathrm{mM} \mathrm{NaCl}$ at $65^{\circ} \mathrm{C}$ for $4 \mathrm{~h}$. The DNA was then treated with proteinase $\mathrm{K}$ (Millipore) at $45^{\circ} \mathrm{C}$ for $1 \mathrm{~h}$ and precipitated chromatin was used as the template for PCR with the primers listed in Table 2.

\section{Statistics}

Each experiment was repeated at least 3 times. Results of experiments are presented as means \pm standard error of the mean. Statistical analyses were performed with the Student's $t$-test (paired and 2-tailed) or a oneway ANOVA with significance declared at $P<0.05$. 

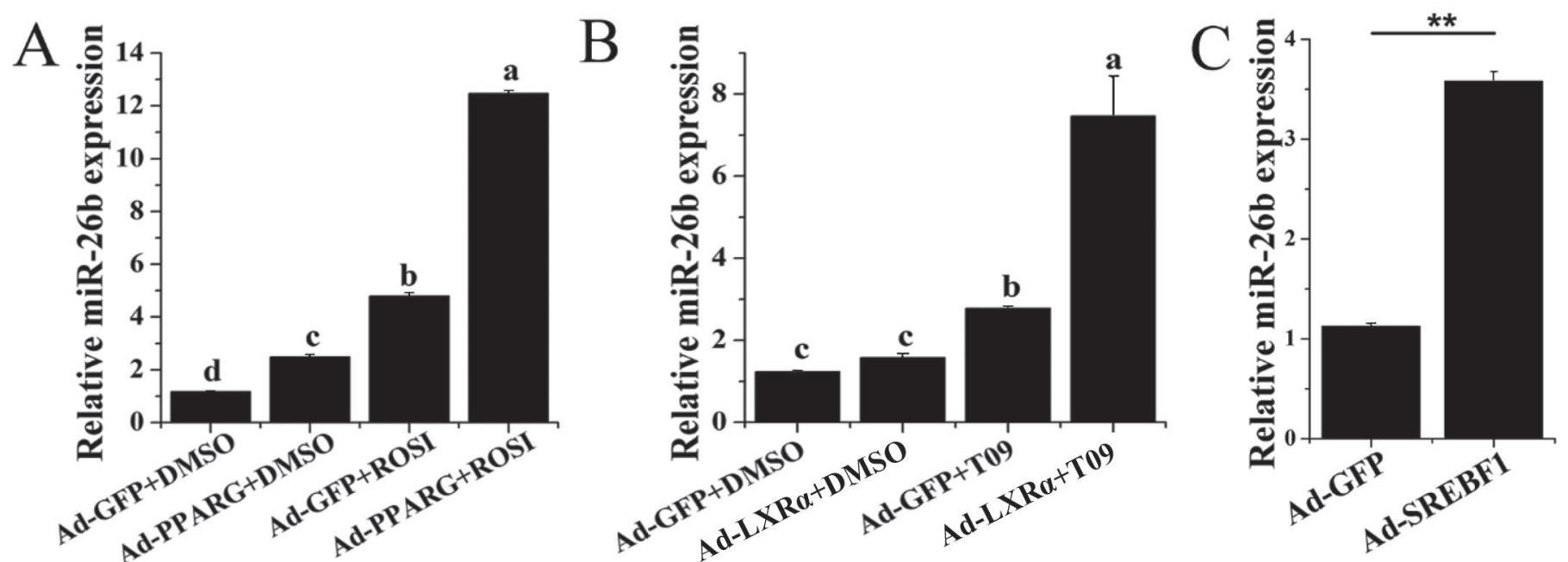

Figure 1. Effect of overexpression of peroxisome proliferator-activated receptor gamma (PPARG), liver X receptor $\alpha$ (LXR $\alpha$ ), or sterol regulatory element binding transcription factor 1 (SREBF1) on miR-26b expression. Cells were treated with (A) adenovirus containing PPARG (Ad-PPARG) + rosiglitazone (ROSI), (B) LXR $\alpha$ + T0901317 (a specific agonist of LXR: T09), or (C) adenovirus containing SREBF1 (AdSREBF1) for $48 \mathrm{~h}$ after which they were harvested for total RNA extraction. The miR-26b expression levels were quantified by quantitative real-time PCR. Each treatment was carried out in triplicate and repeated 3 times $(n=9)$. Values are presented as means \pm SEM. Different letters $(\mathrm{a}-\mathrm{d})$ indicate a significant difference among different treatments. ${ }^{*} * P<0.01$. DMSO $=$ dimethyl sulfoxide. Ad-GFP $=$ adenovirus containing green fluorescent protein.

\section{RESULTS}

\section{MiR-26b Expression Is Regulated by LXRa, PPARG, and SREBF1}

We aimed to study the effects of some lipid-related transcription factors on miR-26b expression profile. As LXR $\alpha$, PPARG, and SREBF1 appear to be key transcription regulators in goat mammary gland lipid metabolism, Ad-PPARG and PPARG agonist-ROSI (Figure 1A), Ad-LXR $\alpha$ and LXR $\alpha$ agonist-T0901317 (Figure 1B), and Ad-SREBF1 (Figure 1C) in GMEC were used to evaluate effects on miR-26b expression. Compared with the control, the expression of miR-26b was significantly increased after the agonist (Figure $1 \mathrm{~A}$ and $1 \mathrm{~B}$ ) or adenovirus treatment (Figure $1 \mathrm{~A}$ and 1C). Furthermore, induction of miR-26b over control was much higher when the adenovirus was co-treated together with the agonist (Figure 1A and 1B). These results indicated that the expression of miR-26b was directly or indirectly regulated by these 3 lipid-related transcription factors.

\section{Cloning and Characterization of the Goat miR-26b Promoter}

MiR-26b is an intronic miRNA located in the intron 3 region of $C T D S P 1$, and our previous study revealed that miR-26b functions synergistically with CTDSP1 to regulate fatty acid synthesis in GMEC. To our knowledge, no research is available on the regulation of miR-26b expression. This prompted us to clone and analyze the $5^{\prime}$-flanking sequence of $C T D S P 1$. To this end, a 1,102-bp fragment of 5 '-flanking sequence containing $1,018 \mathrm{bp}$ upstream of the transcription start sites (TSS; +1) was amplified by PCR (Supplemental Figure S1; https://doi.org/10.3168/jds.2016-12440). An initiation codon ATG was located in exon 1 of the miR-26b host gene CTDSP1. In silico analysis revealed

Table 2. Primers used for bisulfite sequencing PCR (BSP) and chromatin immunoprecipitation (ChIP) assays ${ }^{1}$

\begin{tabular}{lllr}
\hline Primer name & Primer & Primer sequence $\left(5^{\prime}\right.$ to $\left.3^{\prime}\right)$ & Binding region $(\mathrm{bp})$ \\
\hline BSP primer & BSP-F & TTGGAATTTTTGTATTTTATAGTTT & -490 \\
& BSP-R & AAAATAAATCTTCTCTCCAATAATC & -319 \\
ChIP primer & Chip-SRE1-F & GTGTTATCTCCATTTCCAAATG & -304 \\
& Chip-SRE1-R & CCCGCCTCCCTGGCCGCCGAG & -2 \\
& Chip-SRE3-F & ATGGGGAGTGGACGTGGCCGGG & -557 \\
& Chip-SRE3-R & AGTGGGTCTTCTCTCCAGTGGTC & -318 \\
\hline
\end{tabular}

${ }^{1}$ Number of nucleotides upstream (-) from the transcriptional start site. The BSP primers and ChIP primers were used for BSP and ChIP assays, respectively. $\mathrm{F}=$ forward; $\mathrm{R}=$ reverse. 


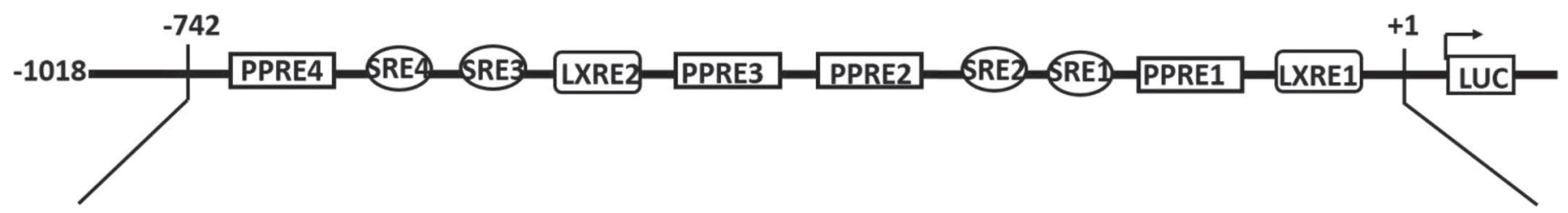

-742 CCGGCGGGTGGGAAGCAGAGGAGGGGGCATGGGAAAAAGGAGGGTTTCATTTCTTCGAGGTCGGTGGGAT PPRE4

-672 TTATCTATGCAAAGAGGTAGGAAGCCTATTCAGGCAGACCGAACAGCACGTATAAAGGCCCTGAGGCGTGGAG

-599 GCCCTCGGCTTCAGGCAAGAAAACCAGGTGGCTGGGGTGGGCATGGGGAGTGGACGTGGCCGGGCGCCCG

-529 GTGAGGAGAGGTCGGCAGGGGCACCCCGGGGCGGACAGCTGGAACTTCTGCACCCTACAGCTCCGGGITTA SRE3 LXRE2

-458 GGGGAAAGGGGCCGAGACTGCGGGTAGGTCACAGTCCGGTTAGGGACCTAATTGTGTGGATCGGCACAGTC PPRE3 PPRE2

-387 GTTTGGAAGTGCAGCAGAGGCTTTGCACGCTCACGACTTCTCCTGCGACCACTGGAGAGAAGACCCACTTT $\underline{\text { TC }}$

-314 AGACCAGATGGTGTTATCTCCATTTCCCAAATGAGAAGACCGAGAAGCTGCCAGTCCCCGACCTTTGGAATGTT SRE2 SRE1

-240 CAGAGGTGGAGTCGCGCTGCCTCGGGGCACTCCCGGGCGCGGGCGCGGGCGCCCCGGGCGGGCCCTAGTCC PPRE1 LXRE1

-169 CGGGCCGCTCAGCGCGGAGAACCGTGGGCCGGGATGCCGGCCCTGCCACGCAGCCGCAGAGGCTTGGCTTC

-98 GCTGGCGAGCTGGCTGGCTGGCGGGCGGCGGGAAAGGAGGCGGCGCCGCCGGGAGCGCGGCAGGAACCT

-29 GGCCGGGCCCGCCTCCCTGGCCGCCGAGCÁ SP1

SP1

TSS

Figure 2. Schematic representation and characterization of the goat miR-26b promoter. +1 indicates the transcription start site (TSS) and is marked by bent arrows, nucleotides of the putative transcription factor binding sites are in boldface, italics, and underlined, and the names are indicated below the sequence. $\mathrm{PPRE}=$ peroxisome proliferator response element; $\mathrm{SRE}=$ sterol regulatory element; LXRE $=$ liver $\mathrm{X}$ receptor $\alpha$ binding elements; $\mathrm{SP} 1=$ specificity protein $1 ; \mathrm{LUC}=$ luciferase.

transcription factor binding sites for SREBP-1 (SRE, $-269,-316,-529$, and $-568 \mathrm{bp})$, LXR (LXRE, -220 and $-515 \mathrm{bp})$, PPARG (PPRE, $-239,-396,-461$, and $-728 \mathrm{bp}$ ), and Sp1 ( -24 and $-63 \mathrm{bp}$; Figure 2).

\section{Effect of LXRa, PPARG, and SREBF1 on the Promoter Methylation of the miR-26b Gene}

Based on the above results, it was concluded that miR-26b is regulated by lipid-related transcription factors. To elucidate the mechanism underlying the upregulation of the miR-26b, we used bisulfite sequencing BSP in GMEC after treatment with Ad-LXR $\alpha+$ T09/Ad-PPARG + ROSI/Ad-SREBF1 to determine whether the expression of miR-26b were regulated by altering the methylation of its promoter. Compared with the control group (Figure 3), BSP results demonstrated a significant decrease in average miR-26b promoter methylation level in GMEC after treatment with Ad-LXR $\alpha+$ T09 (mean of 47.8 vs. $52.2 \%$ ), Ad-PPARG + ROSI (mean of 44.4 vs. 52.2\%), and Ad-SREBF1 (mean of 38.9 vs. $52.2 \%$ ). These results indicated that the methylation level of miR-26b promoter region can be epigenetically regulated by these transcript factors in GMEC.

\section{PPARG Induced the Expression of PPRE-Containing miR-26b Promoter}

In silico analysis revealed that the promoter of miR26b contains 4 PPRE (based on their distance from TSS, the 4 PPRE are distinguished as PPRE1, PPRE2, PPRE3, and PPRE4). To determine if the PPRE in the miR-26b promoter are functional, we cloned a miR$26 \mathrm{~b}$ wild type and PPRE mutation promoters in an inducible reporter system upstream to the firefly luciferase gene. Compared with the wild-type promoters, PPRE1 and PPRE2 mutated alone or simultaneously decreased the promoter activity dramatically (Figure 4A). When GMEC transfected with miR-26b promoter vectors were treated with Ad-PPARG + ROSI, PPRE1 or PPRE2 mutation led to a decrease in the activation effects of Ad-PPARG + ROSI, whereas the activation 
was almost abolished when these 2 sites were mutated simultaneously (Figure 4B). However, a PPRE3 and PPRE4 mutation had no influence on promoter activity (Figure 4A). These results indicated that PPARG is involved in the promoter activity of miR-26b, and the 2 PPRE (PPRE1 and PPRE2) are important for maintaining miR-26b transcriptional activity.

\section{SREBF1 and LXR Induced the Expression of an SRE-Containing miR-26b Promoter}

Bioinformatics analysis revealed 4 SRE (SRE1, SRE2, SRE3, and SRE4) and 2 LXRE (LXRE1 and LXRE2) in the cloning fragment. To identify functional SRE and LXRE elements required for the expression of miR-26b, we constructed a series of site-mutated luciferase reporter vectors and transfected them into GMEC followed by detection of the luciferase activity. Results indicated that single mutation of LXRE and simultaneous mutations of both LXRE did not decrease miR-26b promoter activity significantly (Figure 5A). However, mutations of SRE1 and SRE3 decreased promoter activity dramatically, whereas mutations of SRE2 and SRE4 had no significant influence on promoter activity, and mutations of both SRE1 and SRE3 almost abolished the promoter activity (Figure 5B). These effects were not observed in the SRE2 and SRE4 simultaneous mutation group. When GMEC transfected with miR$26 \mathrm{~b}$ promoter vectors were treated with Ad-SREBF1, the wild type miR-26b promoter increase significantly over the control, and this effect was curtailed when only SRE1 and SRE3 or both of SRE1 and SRE3 were mutated in the miR-26b promoter vectors. Promoter activity was not significantly altered when only the
A

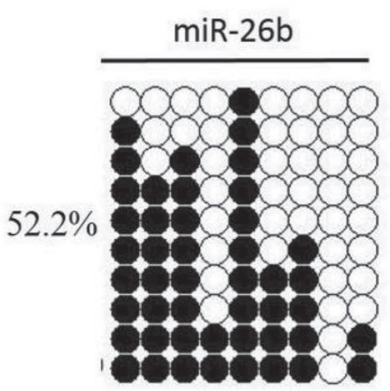

Ad-GFP+DMSO

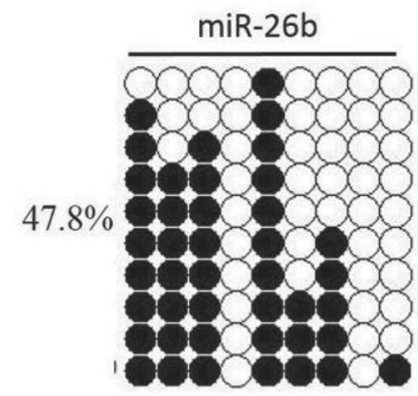

Ad-LXR $\alpha+$ T09

- methylated

$\checkmark$ unmethylated

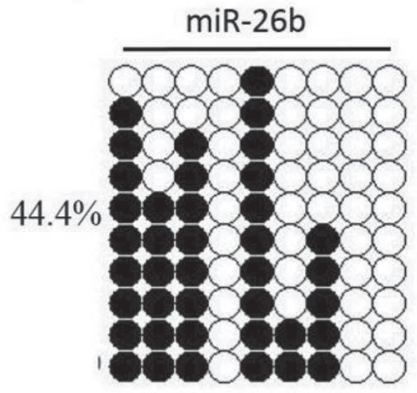

Ad-PPARG+ROSI

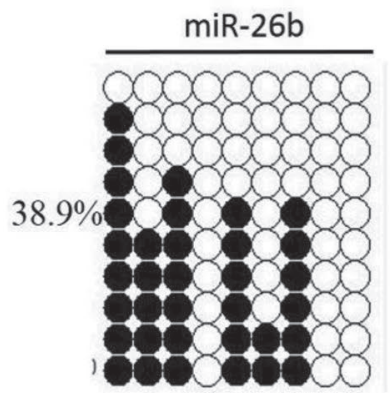

Ad-SREBF 1

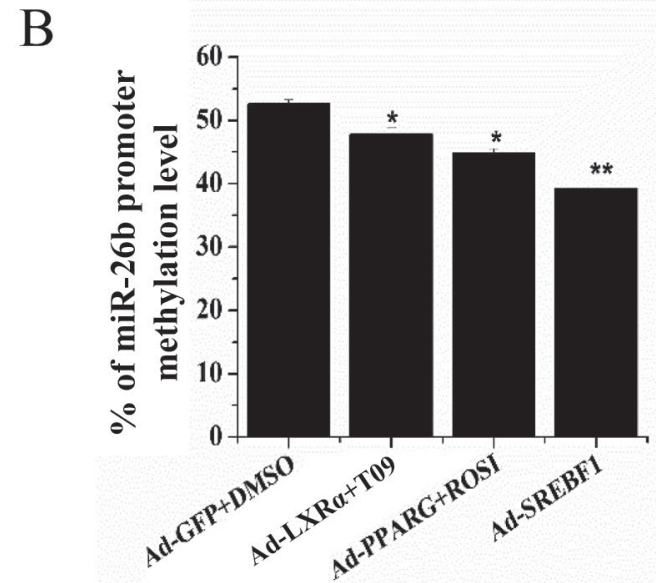

Figure 3. Overexpression of peroxisome proliferator-activated receptor gamma (PPARG), liver X receptor $\alpha$ (LXR $\alpha$ ), or sterol regulatory element binding transcription factor 1 (SREBF1) induce aberrant epigenetic modifications of miR-26b promoter in goat mammary epithelial cells (GMEC). Bisulfite genomic sequencing PCR of miR-26b promoter (A) and methylation ratios (B) in GMEC after the same treatment as in Figure 1. For analysis of the methylation ratios, each treatment was carried out in triplicate and 10 single clones were selected for sequencing for 1 repeat $(\mathrm{n}=30)$. Values are presented as means \pm SEM. ${ }^{*} P<0.05 ;{ }^{* *} P<0.01$. Ad- $=$ adenovirus containing; DMSO $=$ dimethyl sulfoxide; ROSI $=$ rosiglitazone; T09 $=$ T0901317; GFP $=$ green fluorescent protein. 
A
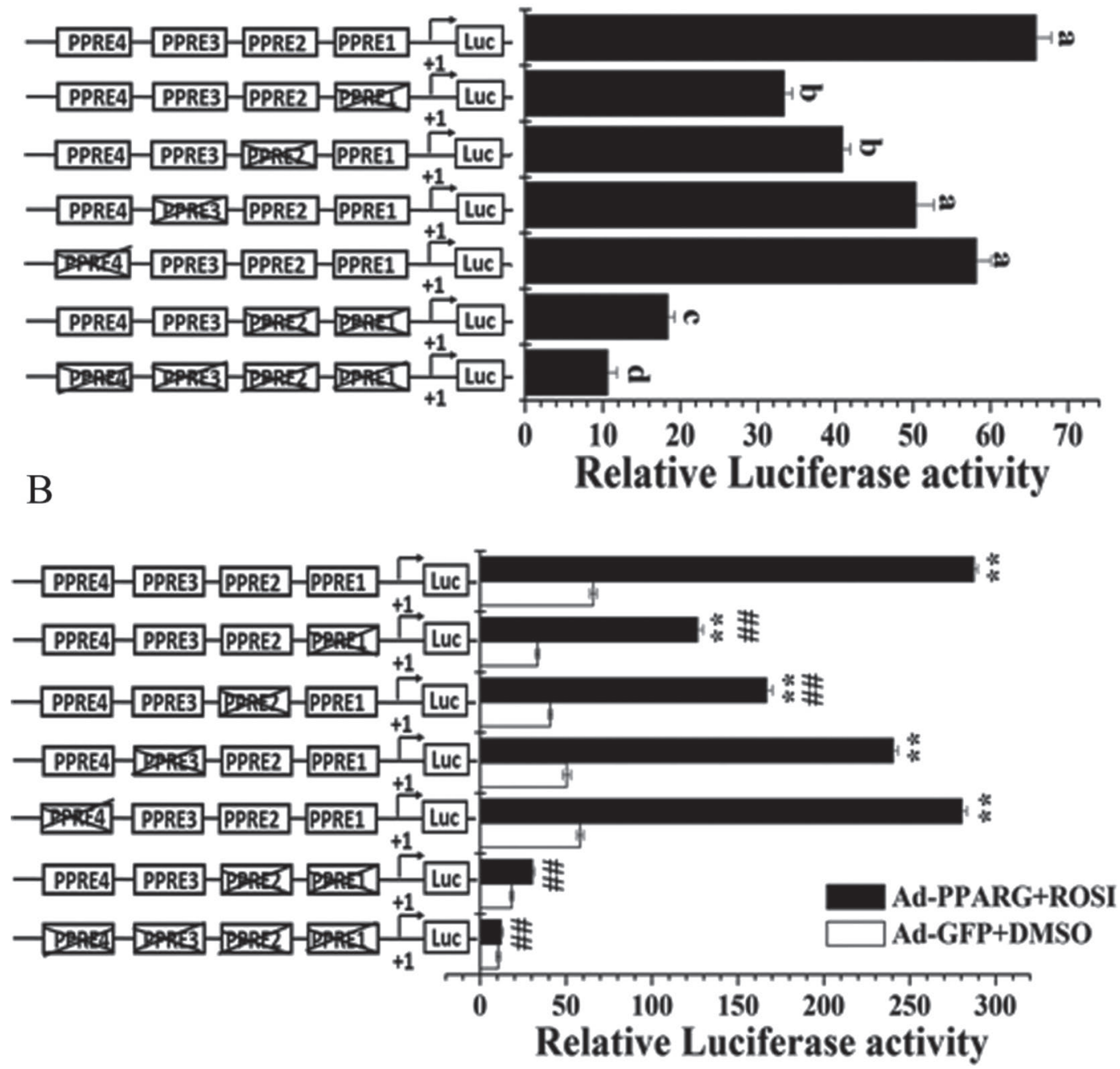

Figure 4. Effects of site-directed mutagenesis of peroxisome proliferator response element (PPRE) on miR-26b promoter activity. (A) Relative luciferase activity changes after $48 \mathrm{~h}$ of transfection with the wild-type and PPRE mutated constructs. (B) Mutation of PPRE in the goat miR-26b promoter suppressed transcription activity in response to peroxisome proliferator-activated receptor gamma (PPARG) overexpression. Each treatment was carried out in triplicate and repeated 3 times $(n=9)$. Values are presented as means \pm SEM. Different letters $(\mathrm{a}-\mathrm{d})$ indicate a significant difference among different treatments. ${ }^{*} P<0.01$ vs. adenovirus containing green fluorescent protein $($ Ad-GFP) + dimethyl sulfoxide (DMSO); \#\#P $<0.01$ vs. wild type constructs. $\mathrm{LUC}=$ luciferase; $\mathrm{ROSI}=$ rosiglitazone.

SRE2 or SRE4 sites were mutated. These results indicated that SRE1 and SRE3 are critical for maintaining miR-26b basal transcriptional activity.

To explore the regulatory mechanisms of LXR $\alpha$ on miR-26b, we transfected SRE and LXRE mutation vector into the GMEC and then treated cells with AdLXR $\alpha+$ T09. When LXRE binding sites were mutated alone or simultaneously, Ad-LXR $\alpha+$ T09 increased the promoter activity significantly (Figure 5D). In contrast, mutation constructs of SRE1 or SRE3 significantly decreased the activity (Figure 5E) and the simultaneous mutation of SRE1 and SRE3 completely abolished the effects of Ad-LXR $\alpha+$ T09 (Figure 5E). These data suggested that LXR participates in the regulation of the miR-26b promoter via an SRE binding site, without the 2 LXRE having an influence on miR-26b promoter activity.

\section{SREBF Binds to the miR-26b Promoter Region In Vivo}

To determine whether the transcription factors SREBF1 binds to the 2 SRE (SRE1 and SRE3) in the miR-26b promoter region in vivo, ChIP was performed in GMEC. Chromatin was immunoprecipitated with an SREBF1 antibody, and DNA fragments of the expected 
A

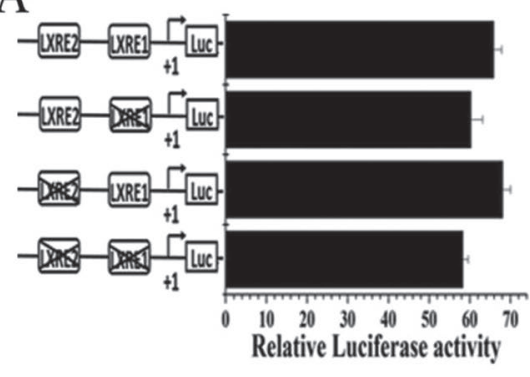

$\mathrm{C}$

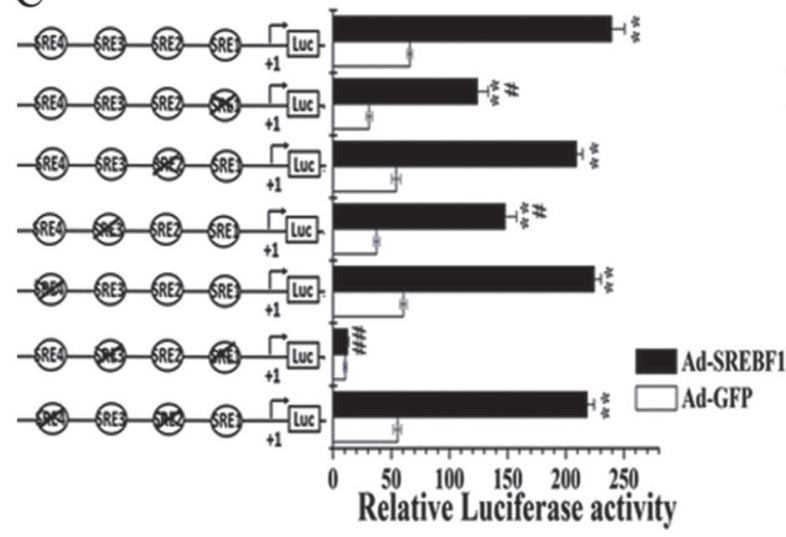

E

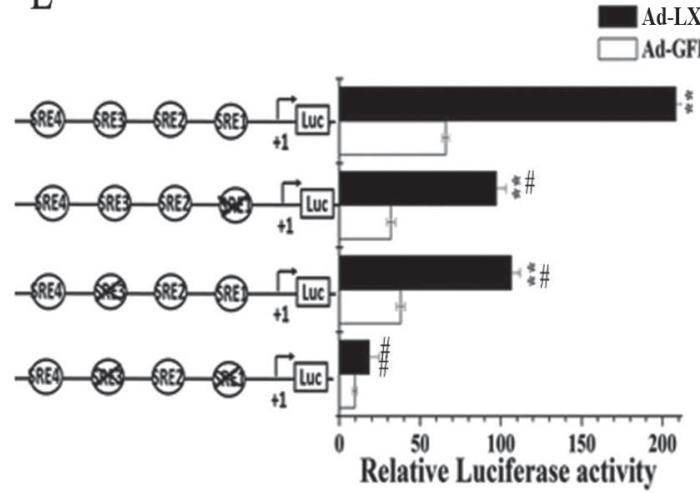

A-LXRa-T09 d-GFP+DMSO
$\mathrm{B}$

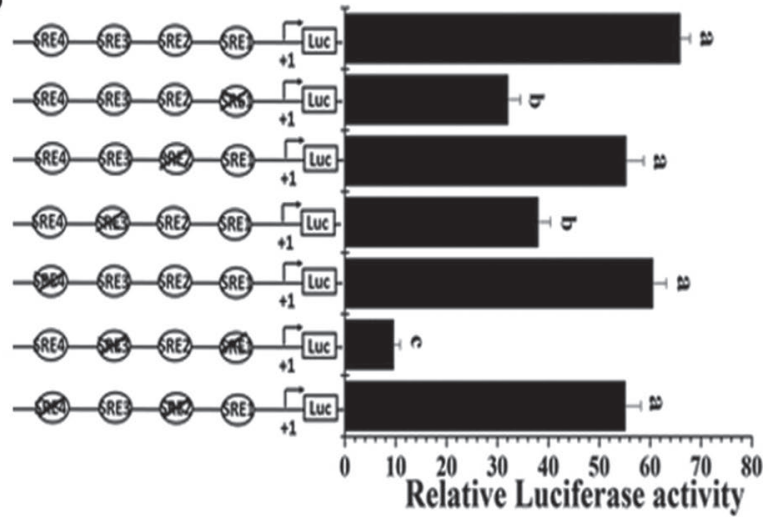

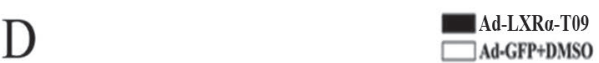
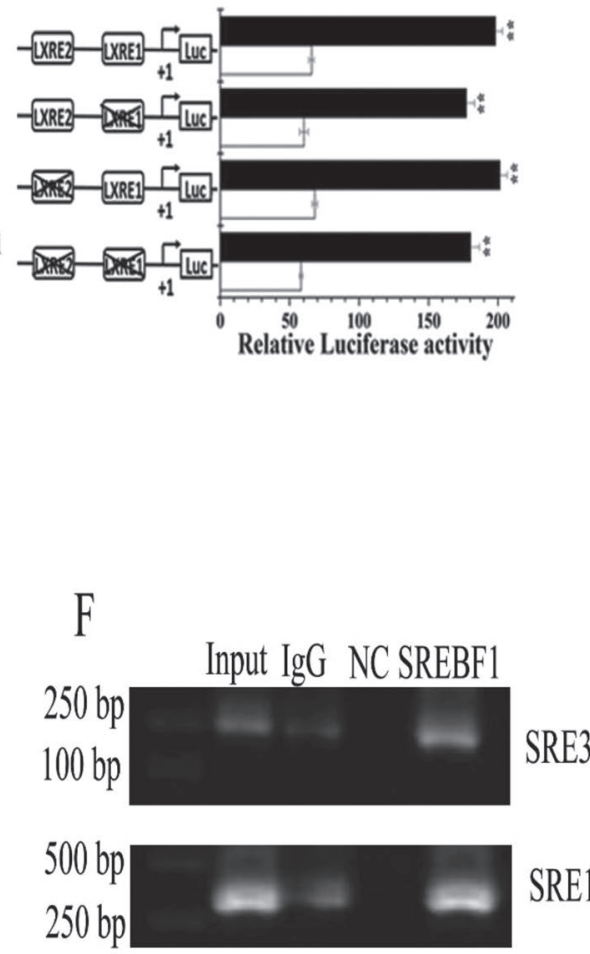

Figure 5. Effects of site-directed mutagenesis of LXR response element (LXRE) and sterol response element (SRE) on miR-26b promoter activity. Relative luciferase activity changes after $48 \mathrm{~h}$ of transfection with the wild-type and (A) LXRE mutated constructs or (B) SRE mutated constructs. (C) Mutation of SRE in the goat miR-26b promoter suppressed transcriptional activity in response to sterol regulatory element binding transcription factor 1 (SREBF1) overexpression. (D) Mutation of LXRE in the goat miR-26b promoter had no influence on transcriptional activity in response to liver X receptor $\alpha(\mathrm{LXR} \alpha)$ overexpression. (E) Mutation of SRE in the goat miR-26b promoter decreased transcriptional activity in response to LXR $\alpha$ overexpression. Each treatment was carried out in triplicate and repeated 3 times $(n=9)$. (F) Binding of SREBF1 to the miR-26b promoter region as analyzed by ChIP; DNA isolated from immunoprecipitated material was amplified by PCR targeting the SRE1 (303 bp) and SRE3 (239 bp) sequences. Total chromatin was used as the input; a normal IgG was used as a negative control. NC = no antibody was used; LUC = luciferase; T09 $=$ T0901317. Values are presented as means \pm SEM. Different letters (a-c) indicate a significant difference among different treatments. ${ }^{* *} P<0.01$ vs. adenovirus containing green fluorescent protein(Ad-GFP) + dimethyl sulfoxide (DMSO)/ Ad-GFP; $\# P<0.05$, \#\#P $<0.01$ vs. wild type constructs.

size were used as a template for PCR amplification. The ChIP assay showed that SREBF1 interacted with the miR-26b promoter at the SRE1 and SRE3 sites
(Figure $5 \mathrm{~F}$ ), indicating that the -258 to $-269 \mathrm{bp}$ and -519 to -529 bp regions in the miR-26b promoter can bind to the SREBF1 transcription factor. 


\section{DISCUSSION}

Previous studies revealed that the miR-26b and its direct target ADAM metallopeptidase domain 17 are the key players in human adipogenesis and drive characteristics of brown adipocytes (Karbiener et al., 2014). In addition, miR-26b and its host gene CTDSP1 synergistically blocked the G1/S transition by activating $\mathrm{pRb}$ (Zhu et al., 2012). Our previous study revealed the miR-26b and CTDSP1 synergistically regulated the synthesis of UFA and triacylglycerol by suppressing the expression of INSIG1 in goat mammary cells (Wang et al., 2016).

Although miR-26b has been widely studied in numerous biogenesis, for instance neuronal differentiation (Dill et al., 2012), cell cycle transition (Zhu et al., 2012), and triacylglycerol synthesis (Wang et al., 2016). To our knowledge, no research is available on the regulation of miR-26b expression. Currently, it is believed that miR-26b is transcribed along with CTDSP1, as the nearest significant upstream PolIIChIP-chip peak region overlapped the $5^{\prime}$ region of their host gene (Corcoran et al., 2009), suggesting that miR-26b and $C T D S P 1$ share the same TSS. It is known that the genomic regions immediately upstream of the TSS of protein coding genes exhibit high levels of sequence conservation (Carninci et al., 2005; Taylor et al., 2006), which is probably related to the high concentration of cis-regulatory sites in this region (Prestridge and Burks, 1993). Based on this available information regarding the fact that TSS of intragenic miR-26b overlaps its host gene's TSS, we cloned the 5 ' flanking region of the goat miR-26b gene that contains the typical promoter characters such as GC islands and transcription factorbinding sites. Furthermore, cells were transfected with this $5^{\prime}$ flanking region exhibited a significant increase in promoter activity underscoring an important role for this genomic region and the likelihood that it contains promoter function.

Understanding the regulatory mechanisms of key lipid-related transcription factors on miR-26b may give insights into mechanism whereby miRNA could control lipid metabolism in mammals. This prompted us to probe which transcription factors might regulate miR26b gene expression through a promoter region by analyzing genomic databases of the ENCODE project with the UCSC genome Brower. This analysis predicted the presence of binding sites for transcription factors such as SP1, LXR $\alpha$, PPARG, and SREBF1 among others. Theses transcription factors have well-known functions in lipid metabolism and adipose tissue function. Thus, the presence of these binding sites in the putative miR$26 \mathrm{~b}$ promoter supports available data indicating that the miR-26b is regulated by key lipid-related nuclear receptors.

The SREBP are basic helix-loop-helix leucine zipper transcription factors that are synthetized as inactive precursors anchored in the membrane of the endoplasmic reticulum (Horton et al., 2002; Eberlé et al., 2004). Upon activation, the precursor protein undergoes 2 sequential steps of proteolytic processing in the Golgi apparatus, resulting in the release of the $N$-terminal active fragment (mature SREBP protein). Mature SREBP then translocate to the nucleus and bind to SRE in the promoter of target genes to regulate lipid metabolism (Horton et al., 2002; Xu et al., 2016). In our present studies, we observed that upregulation of SREBF1 triggered miR-26b expression, a result in accordance with the miR-26b promoter showing lower promoter activity when the SRE were mutated.

Another important upstream regulator that modulates lipid metabolism is LXR (McFadden and Corl, 2010; Matsushita et al., 2016). The LXR $\alpha$ and LXR $\beta$ are ligand-activated nuclear receptors that can form heterodimers with retinoid X receptors (RXR) in response to synthetic agonists such as T09 (Liang et al., 2004; Fan et al., 2015), upon which the complex can bind to specific DNA sequences (known as LXRE) in the promoter of target genes (Yoshikawa et al., 2001). The LXRE generally consists of 2 direct repeat (DR4-type element) half-sites of a consensus hexameric sequence (AGGTCA), also defined as a degenerate DR4-type element containing nucleotide substitutions in both half-sites that deviate from the canonical AGGTCA motif (Willy and Mangelsdorf, 1997). The LXR $\beta$ is ubiquitously expressed and its expression is lower than LXR $\alpha$, and the LXR $\alpha$ is predominantly restricted to lipogenic tissues such as liver and adipose tissue (Vinod et al., 2014), combined with the fact that the expression of $L X R \alpha$ was higher in lactation than the nonlactation in bovine, indicating that LXR $\alpha$, not LXR $\beta$, plays an important role in the control of lipid metabolism in mammary gland. Therefore, we selected LXR $\alpha$ to explore a potential regulatory mechanism over miR-26b at the transcriptional level. Although the bioinformatics analysis results indicated 2 LXRE sites in the promoter of miR-26b, the 2 potential LXRE complexes did not induce a response to the LXR activation. This may be due to the 2 putative LXRE, which are not conserved DR4 elements. Another possible explanation is that the predictive binding sites obtained from bioinformatics analyses were not accurate. In our experiments, the fact that the promoter activity of miR-26b was not changed after activation of $L X R \alpha$ combined with the fact that site-mutated SRE markedly decreased the LXR $\alpha$-induced promoter activation 
suggests that LXR $\alpha$ regulates miR-26b transcription mainly through an indirect interaction by inducing the expression of SREBP1 and SRE binding sites.

The PPAR are ligand-activated intracellular transcription factors, members of the nuclear hormone receptor superfamily (Gervois et al., 2000; Tontonoz and Spiegelman, 2008). The PPAR subfamily consists of 3 subtypes encoded by distinct genes denoted as $P P A R \alpha, P P A R \beta / \delta$, and PPAR $\gamma$, which are activated by selective ligands. The PPAR $\gamma$ contains one nuclear receptor DNA binding domain and is a receptor that binds peroxisome proliferators such as hypolipidemic drugs and fatty acids. It plays an important role in the regulation of lipid homeostasis, adipogenesis, insulin resistance, and development of various organs. The PPARG always functions as an obligate heterodimer with RXR (Chandra et al., 2008), and the complex transfers to the nucleus and activates PPARG in a ligand-dependent manner on a specific subset of promoters that contain the PPARG binding site (Temple et al., 2005; Lodhi and Semenkovich, 2014).

Previous studies revealed that many lipid metabolism-related genes contains the PPRE sequence ( $\mathrm{Yu}$ et al., 2015; Zhou, 2015), which indicated that PPARG is a key regulator of adipogenesis. Our recent studies revealed that the expression of genes related to triacylglycerol synthesis and secretion were significantly upregulated in GMEC after treatment with ROSI (Shi et al., 2013), and there is a putative PPRE site in the promoter of $A D R P$ (Yu et al., 2015). With bioinformatics and experimental validation, Dharap et al. (2015) reported that promoters of many miRNA contain PPRE and PPARG activation induces those miRNA in mice. However, whether PPARG directly regulates miR-26b is still unknown, especially in ruminants.

In the present study, the mRNA level of miR-26b was dramatically upregulated by ROSI and Ad-PPARG. To explore whether PPARG could regulate the expression of miR-26b gene directly in GMEC, we cloned the goat miR-26b promoter including 4 potential PPRE in the promoter. The site-directed mutagenesis of the PPRE in the miR-26b promoter demonstrated that PPRE1 and PPRE2 are 2 key elements for allowing transcriptional activity induced by PPARG and ROSI. The use of ChIP technology is needed to further verify this proposed molecular mechanism; however, in the present study we could not find a suitable antibody for this assay.

The CTDSP1/miR-26b is located at chromosome $2 \mathrm{q} 35$, and $\mathrm{CpG}$ islands in the promoter regions (between $-2,000$ and $600 \mathrm{bp}$ ) of CTDSP1/miR-26b have been reported (Zhu et al., 2012). Based on this, we hypothesized that promoter methylation may also play a role in controlling miR-26b expression in GMEC. In addition, the $\mathrm{CpG}$ islands in the miR-26b promoter region have some putative PPARG, SREBF1 binding sites. To evaluate whether overexpression of these transcription factors has an effect on the methylation of miR-26b, the BSP assay was performed. The fact that the percentage of methylation of $\mathrm{CpG}$ sites in transcription factors activation group was lower than the control group, combined with the fact that these transcription factors can directly or indirectly bind to cis-regulatory elements in the promoter of miR-26b, suggested that miR-26b is synergistically regulated by these 2 mechanisms.

\section{CONCLUSIONS}

In the present study, we cloned a 1,018-bp fragment of the $5^{\prime}$ upstream region of the miR-26b gene TSS site that exhibits promoter activity in GMEC. Furthermore, according to luciferase reporter assays, transcription activity through the promoter was upregulated by PPARG, SREBP, and LXR $\alpha$. The SRE and PPRE elements characterized allowed for deciphering a direct role of SREBP1 in maintaining miR-26b expression, and also that LXR $\alpha$ promotes miR-26b expression in an SREBP-1-dependent manner. These transcription factors also can regulate miR-26b expression through DNA methylation. Altogether, these findings contribute to better understanding of the interaction between precise promoter elements that control the transcription and translation of miR-26b genes. However, further research should be done to identify the functional cis-elements within the miR-26b promoter and to study specific regulatory mechanisms of miR-26b and its role in lipid metabolism.

\section{ACKNOWLEDGMENTS}

This research was jointly supported by the National Natural Science Foundation of China (Beijing), Special Fund for Agro-Scientific Research in the Public Interest (Beijing), and the Transgenic New Species Breeding Program of China (Beijing); contract grant numbers: no. 31372281, no. 201103038, and no. 2014ZX08009051B. The authors declare no conflicts of interest.

\section{REFERENCES}

Baskerville, S., and D. P. Bartel. 2005. Microarray profiling of microRNAs reveals frequent coexpression with neighboring miRNAs and host genes. RNA 11:241-247.

Carninci, P., T. Kasukawa, S. Katayama, J. Gough, M. C. Frith, N Maeda, R. Oyama, T. Ravasi, B. Lenhard, C. Wells, R. Kodzius, K. Shimokawa, V. B. Bajic, S. E. Brenner, S. Batalov, A. R. Forrest, M. Zavolan, M. J. Davis, L. G. Wilming, V. Aidinis, J. E. Allen, A. Ambesi-Impiombato, R. Apweiler, R. N. Aturaliya, T. L. 
Bailey, M. Bansal, L. Baxter, K. W. Beisel, T. Bersano, H. Bono, A. M. Chalk, K. P. Chiu, V. Choudhary, A. Christoffels, D. R. Clutterbuck, M. L. Crowe, E. Dalla, B. P. Dalrymple, B. de Bono, G. Della Gatta, D. di Bernardo, T. Down, P. Engstrom, M. Fagiolini, G. Faulkner, C. F. Fletcher, T. Fukushima, M. Furuno, S. Futaki, M. Gariboldi, P. Georgii-Hemming, T. R. Gingeras, T. Gojobori, R. E. Green, S. Gustincich, M. Harbers, Y. Hayashi, T. K. Hensch, N. Hirokawa, D. Hill, L. Huminiecki, M. Iacono, K. Ikeo, A. Iwama, T. Ishikawa, M. Jakt, A. Kanapin, M. Katoh, Y. Kawasawa, J. Kelso, H. Kitamura, H. Kitano, G. Kollias, S P. Krishnan, A. Kruger, S. K. Kummerfeld, I. V. Kurochkin, L. F. Lareau, D. Lazarevic, L. Lipovich, J. Liu, S. Liuni, S. McWilliam, M. Madan Babu, M. Madera, L. Marchionni, H. Matsuda, S. Matsuzawa, H. Miki, F. Mignone, S. Miyake, K. Morris, S. Mottagui-Tabar, N. Mulder, N. Nakano, H. Nakauchi, P. Ng, R. Nilsson, S. Nishiguchi, S. Nishikawa, F. Nori, O. Ohara, Y. Okazaki, V. Orlando, K. C. Pang, W. J. Pavan, G. Pavesi, G. Pesole, N. Petrovsky, S. Piazza, J. Reed, J. F. Reid, B. Z. Ring, M. Ringwald, B. Rost, Y. Ruan, S. L. Salzberg, A. Sandelin, C. Schneider, C. Schönbach, K. Sekiguchi, C. A. Semple, S. Seno, L. Sessa, Y. Sheng, Y Shibata, H. Shimada, K. Shimada, D. Silva, B. Sinclair, S. Sperling, E. Stupka, K. Sugiura, R. Sultana, Y. Takenaka, K. Taki, K. Tammoja, S. L. Tan, S. Tang, M. S. Taylor, J. Tegner, S. A. Teichmann, H. R. Ueda, E. van Nimwegen, R. Verardo, C. L. Wei, K. Yagi, H. Yamanishi, E. Zabarovsky, S. Zhu, A. Zimmer, W. Hide, C. Bult, S. M. Grimmond, R. D. Teasdale, E. T. Liu, V. Brusic, J. Quackenbush, C. Wahlestedt, J. S. Mattick, D. A. Hume, C. Kai, D. Sasaki, Y. Tomaru, S. Fukuda, M. Kanamori-Katayama, M. Suzuki, J. Aoki, T. Arakawa, J. Iida, K. Imamura, M. Itoh, T. Kato, H. Kawaji, N. Kawagashira, T. Kawashima, M. Kojima, S. Kondo, H. Konno, K. Nakano, N. Ninomiya, T. Nishio, M. Okada, C. Plessy, K. Shibata, T. Shiraki, S. Suzuki, M. Tagami, K. Waki, A. Watahiki, Y. Okamura-Oho, H. Suzuki, J. Kawai, Y. Hayashizaki, FANTOM Consortium, and RIKEN Genome Exploration Research Group and Genome Science Group (Genome Network Project Core Group). 2005. The transcriptional landscape of the mammalian genome. Science 309:1559-1563.

Chandra, V., P. Huang, Y. Hamuro, S. Raghuram, Y. Wang, T. P. Burris, and F. Rastinejad. 2008. Structure of the intact PPAR- $\gamma$ RXR- $\alpha$ nuclear receptor complex on DNA. Nature 456:350-356.

Corcoran, D. L., K. V. Pandit, B. Gordon, A. Bhattacharjee, N. Kaminski, and P. V. Benos. 2009. Features of mammalian microRNA promoters emerge from polymerase II chromatin immunoprecipitation data. PLoS One 4:e5279.

Daimiel-Ruiz, L., M. Klett-Mingo, V. Konstantinidou, V. Mico, J. F. Aranda, B. Garcia, J. Martinez-Botas, A. Davalos, C. FernandezHernando, and J. M. Ordovas. 2015. Dietary lipids modulate the expression of miR-107, an miRNA that regulates the circadian system. Mol. Nutr. Food Res. 59:552-565.

Dharap, A., C. Pokrzywa, S. Murali, B. Kaimal, and R. Vemuganti. 2015. Mutual induction of transcription factor PPARgamma and microRNAs miR-145 and miR-329. J. Neurochem. 135:139-146.

Dill, H., B. Linder, A. Fehr, and U. Fischer. 2012. Intronic miR-26b controls neuronal differentiation by repressing its host transcript, ctdsp2. Genes Dev. 26:25-30.

Eberlé, D., B. Hegarty, P. Bossard, P. Ferré, and F. Foufelle. 2004. SREBP transcription factors: Master regulators of lipid homeostasis. Biochimie 86:839-848.

Fan, H., W. Dong, Q. Li, X. Zou, Y. Zhang, J. Wang, S. Li, W. Liu, Y. Dong, and H. Sun. 2015. Ajuba preferentially binds LXR $\alpha / R X R \gamma$ heterodimer to enhance LXR target gene expression in liver cells. Mol. Endocrinol. 29:1608-1618.

Fan, Y. M., P. J. Karhunen, M. Levula, E. Ilveskoski, J. Mikkelsson, O. A. Kajander, O. Järvinen, N. Oksala, J. Thusberg, and M. Vihinen. 2008. Expression of sterol regulatory element-binding transcription factor (SREBF) 2 and SREBF cleavage-activating protein (SCAP) in human atheroma and the association of their allelic variants with sudden cardiac death. Thromb. J. 6:17.

Filipowicz, W., S. N. Bhattacharyya, and N. Sonenberg. 2008. Mechanisms of post-transcriptional regulation by microRNAs: Are the answers in sight? Nat. Rev. Genet. 9:102-114.
Fu, X., Z. Meng, W. Liang, Y. Tian, X. Wang, W. Han, G. Lou, X. Wang, F. Lou, and Y. Yen. 2014. miR-26a enhances miRNA biogenesis by targeting Lin28B and Zcchc11 to suppress tumor growth and metastasis. Oncogene 33:4296-4306.

Gervois, P., I. P. Torra, J. C. Fruchart, and B. Staels. 2000. Regulation of lipid and lipoprotein metabolism by PPAR activators. Clin. Chem. Lab. Med. 38:3-11.

Horton, J. D., J. L. Goldstein, and M. S. Brown. 2002. SREBPs: Activators of the complete program of cholesterol and fatty acid synthesis in the liver. J. Clin. Invest. 109:1125-1131.

Kang, K., X. Zhang, H. Liu, Z. Wang, J. Zhong, Z. Huang, X. Peng, Y. Zeng, Y. Wang, Y. Yang, J. Luo, and D. Gou. 2012. A novel real-time PCR assay of microRNAs using S-Poly(T), a specific oligo(dT) reverse transcription primer with excellent sensitivity and specificity. PLoS One 7:e48536.

Karbiener, M., D. F. Pisani, A. Frontini, L. M. Oberreiter, E. Lang, A. Vegiopoulos, K. Mössenböck, G. A. Bernhardt, T. Mayr, F. Hildner, J. Grillari, G. Ailhaud, S. Herzig, S. Cinti, E. Z. Amri, and M. Scheideler. 2014. MicroRNA-26 family is required for human adipogenesis and drives characteristics of brown adipocytes. Stem Cells 32:1578-1590

Kim, V. N., J. Han, and M. C. Siomi. 2009. Biogenesis of small RNAs in animals. Nat. Rev. Mol. Cell Biol. 10:126-139.

Li, J., J. Luo, H. Xu, M. Wang, J. Zhu, H. Shi, A. B. Haile, H. Wang, and Y. Sun. 2015a. Fatty acid synthase promoter: Characterization, and transcriptional regulation by sterol regulatory element binding protein-1 in goat mammary epithelial cells. Gene $561: 157-164$.

Li, J., J. Luo, J. Zhu, Y. Sun, D. Yao, H. Shi, and W. Wang. 2015b. Regulation of the fatty acid synthase promoter by liver $\mathrm{X}$ receptor alpha through direct and indirect mechanisms in goat mammary epithelial cells. Comp. Biochem. Physiol. B Biochem. Mol. Biol. 184:44-51

Liang, Y., S. Lin, T. P. Beyer, Y. Zhang, X. Wu, K. R. Bales, R. B. Demattos, P. C. May, S. D. Li, and X. C. Jiang. 2004. A liver $\mathrm{X}$ receptor and retinoid $\mathrm{X}$ receptor heterodimer mediates apolipoprotein E expression, secretion and cholesterol homeostasis in astrocytes. J. Neurochem. 88:623-634.

Lodhi, I. J., and C. F. Semenkovich. 2014. Peroxisomes: A nexus for lipid metabolism and cellular signaling. Cell Metab. 19:380-392.

Matsushita, K., F. Morello, Z. Zhang, T. Masuda, S. Iwanaga, K. R. Steffensen, J. A. Gustafsson, R. E. Pratt, and V. J. Dzau. 2016 Nuclear hormone receptor LXR $\alpha$ inhibits adipocyte differentiation of mesenchymal stem cells with Wnt/beta-catenin signaling. Lab. Invest. 96:230-238.

McFadden, J. W., and B. A. Corl. 2010. Activation of liver X receptor (LXR) enhances de novo fatty acid synthesis in bovine mammary epithelial cells. J. Dairy Sci. 93:4651-4658.

Monteys, A. M., R. M. Spengler, J. Wan, L. Tecedor, K. A. Lennox, Y. Xing, and B. L. Davidson. 2010. Structure and activity of putative intronic miRNA promoters. RNA 16:495-505.

Prestridge, D. S., and C. Burks. 1993. The density of transcriptional elements in promoter and non-promoter sequences. Hum. Mol. Genet. 2:1449-1453.

Shi, H., J. Luo, J. Zhu, J. Li, Y. Sun, X. Lin, L. Zhang, D. Yao, and H. Shi. 2013. PPAR $\gamma$ regulates genes involved in triacylglycerol synthesis and secretion in mammary gland epithelial cells of dairy goats. PPAR Res. 2013:310948.

Shi, H., H. Shi, J. Luo, W. Wang, A. B. Haile, H. Xu, and J. Li. 2014. Establishment and characterization of a dairy goat mammary epithelial cell line with human telomerase (hT-MECs). Anim. Sci. J. 85:735-743. (Nihon Chikusan Gakkaiho).

Taylor, J., S. Tyekucheva, D. C. King, R. C. Hardison, W. Miller, and F. Chiaromonte. 2006. ESPERR: Learning strong and weak signals in genomic sequence alignments to identify functional elements. Genome Res. 16:1596-1604.

Temple, K. A., R. N. Cohen, S. R. Wondisford, C. Yu, D. Deplewski, and F. E. Wondisford. 2005. An intact DNA-binding domain is not required for peroxisome proliferator-activated receptor gamma (PPARgamma) binding and activation on some PPAR response elements. J. Biol. Chem. 280:3529-3540. 
Tontonoz, P., and B. M. Spiegelman. 2008. Fat and beyond: The diverse biology of PPAR $\gamma$. Annu. Rev. Biochem. 77:289-312.

Vinod, M., I. Chennamsetty, S. Colin, L. Belloy, F. De Paoli, H. Schaider, W. F. Graier, S. Frank, D. Kratky, B. Staels, G. Chinetti-Gbaguidi, and G. M. Kostner. 2014. miR-206 controls LXRo expression and promotes LXR-mediated cholesterol efflux in macrophages. Biochim. Biophys. Acta 1841:827-835.

Wang, D., and H. S. Sul. 1997. Upstream stimulatory factor binding to the E-box at -65 is required for insulin regulation of the fatty acid synthase promoter. J. Biol. Chem. 272:26367-26374.

Wang, H., J. Luo, T. Zhang, H. Tian, Y. Ma, H. Xu, D. Yao, and J. J. Loor. 2016. MicroRNA-26a/b and their host genes synergistically regulate triacylglycerol synthesis by targeting the INSIG1 gene. RNA Biol. 13:500-510.

Wang, W., J. Luo, Y. Zhong, X. Z. Lin, H. B. Shi, J. J. Zhu, J. Li, Y. T. Sun, and W. S. Zhao. 2012. Goat liver X receptor $\alpha$, molecular cloning, functional characterization and regulating fatty acid synthesis in epithelial cells of goat mammary glands. Gene 505:114-120.

Wang, Z., J. Luo, and W. Wang. 2010. Characterization and culture of isolated primary dairy goat mammary gland epithelial cells. Chin. J. Biotechnol. 26:1123-1127.

Willy, P. J., and D. J. Mangelsdorf. 1997. Unique requirements for retinoid-dependent transcriptional activation by the orphan receptor LXR. Genes Dev. 11:289-298.
Xu, H. F., J. Luo, H. Wang, H. Wang, T. Zhang, H. Tian, D. Yao, and J. J. Loor. 2016. Sterol regulatory element binding protein-1 (SREBP-1) c promoter: Characterization and transcriptional regulation by mature SREBP-1 and liver X receptor $\alpha$ in goat mammary epithelial cells. J. Dairy Sci. 99:1595-1604.

Yao, D. W., J. Luo, Q. Y. He, J. Li, H. Wang, H. B. Shi, H. F. Xu, M. Wang, and J. J. Loor. 2016. Characterization of the liver X receptor-dependent regulatory mechanism of goat stearoyl-coenzyme A desaturase 1 gene by linoleic acid. J. Dairy Sci. 99:3945-3957.

Yoshikawa, T., H. Shimano, M. Amemiyakudo, N. Yahagi, A. H. Hasty, T. Matsuzaka, H. Okazaki, Y. Tamura, Y. Iizuka, and K. Ohashi. 2001. Identification of liver $\mathrm{X}$ receptor-retinoid $\mathrm{X}$ receptor as an activator of the sterol regulatory element-binding protein $1 \mathrm{c}$ gene promoter. Mol. Cell. Biol. 21:2991-3000.

Yu, K., H. B. Shi, J. Luo, J. Li, W. S. Zhao, H. B. Tian, and H. P. Shi. 2015. PPARG modulated lipid accumulation in dairy GMEC via regulation of ADRP gene. J. Cell. Biochem. 116:192-201.

Zhou, Y. 2015. Activation of by PPAR $\gamma 1$ through a functional PPRE in transdifferentiation of myoblasts to adipocytes induced by EPA. Cell Cycle 14:1830-1841.

Zhu, Y., Y. Lu, Q. Zhang, J. J. Liu, T. J. Li, J. R. Yang, C. Zeng, and S. M. Zhuang. 2012. MicroRNA-26a/b and their host genes cooperate to inhibit the G1/S transition by activating the $\mathrm{pRb}$ protein. Nucleic Acids Res. 40:4615-4625. 